\title{
Efficiency Enhancement of Organic Solar Cells Using Transparent Plasmonic Ag Nanowire Electrodes
}

\author{
By Myung-Gyu Kang, Ting Xu, Hui Joon Park, Xiangang Luo,* and L. Jay Guo*
}

Organic solar cells (OSCs) offer a promising alternative to inorganic solar cells due to their low cost, easy fabrication, and compatibility with flexible substrates over a large area. Since their first report, ${ }^{[1]}$ the power conversion efficiency (PCE) of OSCs has steadily increased and now reached up to $4-6 \% \cdot{ }^{[2,3]}$ However, further enhancement of the PCE is still required for practical applications. ${ }^{[4]}$ Since the photo-current of the solar cell is directly related to the light intensity, ${ }^{[5]}$ an effective approach to enhance the efficiency of a thin film organic solar cell is to increase the light absorption of the organic film without having to increase the photoactive layer thickness. This constraint mainly comes from the low carrier mobility of organic materials. For this purpose, several light trapping approaches including a folded configuration, ${ }^{[6]}$ diffraction grating, ${ }^{[7]}$ photonic crystal, ${ }^{[8]}$ and surface plasmon resonance $(\mathrm{SPR})^{[9-14]}$ have been investigated and have demonstrated promising results. Among these, the SPR is one of the most promising approaches due to the enhanced optical field associated with metallic nanostructures. Surface plasmons (SP) are surface waves whose electromagnetic field is confined to the vicinity of the metal-dielectric interface. When the condition of the resonance is fulfilled, this confinement leads to an enhancement of the electromagnetic field at the interface and this aspect has been utilized in many near-field SP-based applications, such as SP interference lithography, ${ }^{[15]}$ superlens imaging ${ }^{[16]}$ and surface enhanced Raman scattering. ${ }^{[17]}$ Since the thickness of the organic semiconductors in OSCs is about several tens of nanometer, which coincides with the field decay length of SP in typical dielectric layer, SPR enhancement is naturally suited to increase the optical absorption of the organic semiconductors. The simplest way to resonantly excite SP is to use nanostructures, such as metallic nanoclusters and periodic nanowires. The field enhancement from nanoclusters is highly localized around the nanoclusters and the possible exciton quenching can limit the utility of such nanoclusters in thin film

[*] Dr. M.-G. Kang, Prof. L. J. Guo

Department of Electrical Engineering and Computer Science

The University of Michigan

Ann Arbor, Michigan 48109 (USA)

E-mail: guo@umich.edu

H. J. Park, Prof. L. J. Guo

Macromolecular Science and Engineering

The University of Michigan

Ann Arbor, Michigan 48109 (USA)

T. Xu, Prof. X. Luo

State Key Laboratory of Optical Technologies for Microfabrication

IOE, Chinese Academy of Science

Chengdu 610209 (China)

E-mail: Ixg@ioe.ac.cn

DOI: 10.1002/adma.201001395 organic solar cells. ${ }^{[9]}$ Moreover, even though Ag is an excellent electrical conductor, most of the works using isolated $\mathrm{Ag}$ nanoparticles have to rely on a separate indium tin oxide (ITO) layer as a transparent electrode. Though ITO is the most widely used transparent electrode for organic optoelectronics, it faces several challenges in terms of cost, mechanical and chemical stability for future low-cost, high performance, large-area, and flexible applications. ${ }^{[18-21]}$ Therefore, it would be very attractive if the metallic nanostructures used to excite the SPR effect can simultaneously act as transparent electrodes for the OSCs. Recently, such attempts have been reported using randomly perforated Ag films and periodic Ag gratings, ${ }^{[13,14]}$ but the devices typically showed extremely low PCE, which cannot even compare with devices made using conventional ITO electrode. In general, since the metallic nanostructures themselves also absorb light strongly at the plasmon resonance condition, direct evidence of absolute power efficiency enhancement in such devices is still lacking.

The purpose of this letter is to introduce the design of organic solar cells with a metal film cathode and a metal nanowire anode, where the enhanced external quantum efficiency (EQE) has been observed in the wavelength range predicted by our calculation and simulation. Despite the polarization dependence of the nanowire electrode and its lower transmittance than that of commercial ITO coated glass, we have observed an overall 35\% increase in the PCE in the former as compared with conventional OSCs made using standard ITO electrode under normal unpolarized incident light condition. We discuss in detail the design principle that we exploited to enhance the optical absorption by the organic semiconductor layer.

Previously we have demonstrated large area metallic nanomesh structure as a possible replacement for the transparent ITO electrode used in organic optoelectronics by virtue of its high transparency and electrical conductivity and mechanical flexibility. ${ }^{[22-24]}$ This work exploits a coupled plasmon mode in a metal-dielectric-metal (MIM) sandwich structure where the dielectric layer is composed of the organic semiconductors, and the metallic layers are chosen to be Ag due to its excellent plasmonic properties in the visible range. One metal layer is continuous and act as cathode, and the other is a periodic nanowire structure acting as semi-transparent anode. The periodic nanowire structure is commonly used to couple the incident plane wave to surface plasmon waves. We performed detailed simulations based on rigorous coupled wave analysis (RCWA) ${ }^{[25]}$ to examine the optical absorption enhancement in the periodic Ag nanowire array-based OSCs. Since the wider Ag nanowires would reflect much of the incident light back into the free space, here we only consider the nanowires with small characteristic width about $55 \mathrm{~nm}$. The organic materials on top of the 40nm-thick periodic Ag nanowire consist of poly(3,4-ethylenedioxythiophene) : 


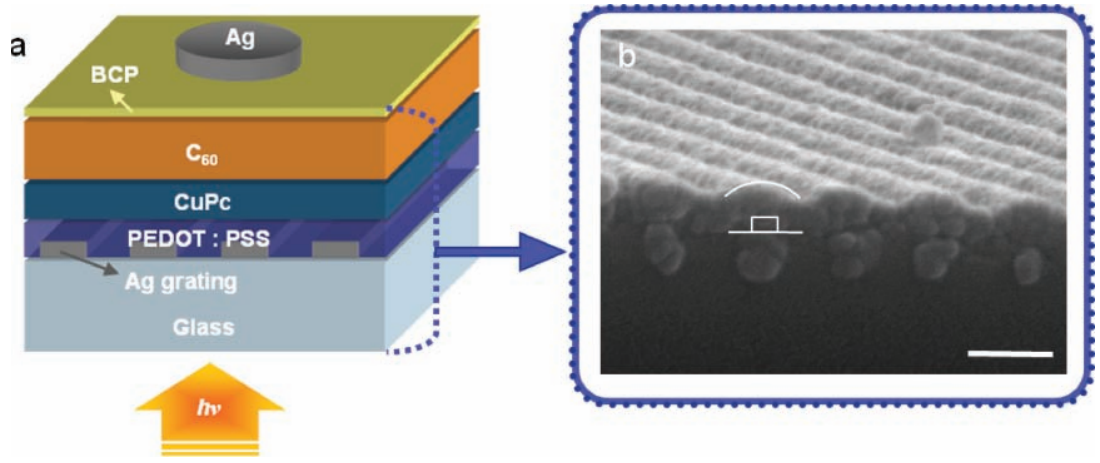

Figure 1. (a) Schematic of the fabricated small molecular weight organic solar cell. The fabricated devices have the layered structure of Ag nanowire anode, PEDOT:PSS, CuPc, C60, BCP, and cathode (thick Ag film) from bottom to top. (b) Cross-sectional view of one of the fabricated devices, but without $70 \mathrm{~nm}$ thick Ag cathode. Organic materials deposited has the sinusoidal shape due to the height profile of the Ag nanowire array and the nature of thermal evaporation process. Organic layers are depicted with solid lines and the square shows the position of the Ag nanowire on glass substrate. The scale bar is $200 \mathrm{~nm}$. is shown as the purple and red dashed lines in Figure 2a. We can see that the absorption enhancement distribution matches well with the calculated plasmon dispersion, which proves our viewpoint. It is also interesting to note that there is another enhancement region for TM polarization at the wavelength greater than $750 \mathrm{~nm}$ that stretches over a board range of grating periods. This feature can be ascribed to the SP resonance with individual nanowire that has been theoretically investigated in Reference 27. Unlike the TM case, the absorption enhancement of TE illumination comes from the conventional MIM waveguide modes: the incident light with TE polarization is scattered by the nanowire array, and coupled to the propagating mode along the waveguide that is bound by the $\mathrm{Ag}$ anode and the Ag cathode. Since the sunlight poly(styrenesulfonate) (PEDOT:PSS, $30 \mathrm{~nm}$ ), copper phthalocyanine (CuPc, $16 \mathrm{~nm}$ ), buckminsterfullerene $\left(\mathrm{C}_{60}, 26 \mathrm{~nm}\right)$, and bathocuproine $(\mathrm{BCP}, 8 \mathrm{~nm})$, which are commonly used as hole transporting layer, electron donor and acceptor layer, and exciton blocking layer, respectively, as shown in Figure 1a. The layer thicknesses correspond to those used in our device fabrication. The simulation also took into account the waviness of the layered structure as seen in the real fabricated devices (Figure 1b).

We calculated the absorption by the organic semiconductor layers by using the absorption coefficients of each organic semiconductor and the optical field distribution in both Ag nanowire device and ITO control device; and define the enhancement factor as the ratio between the two. Figure $2 \mathrm{a}$ and $2 \mathrm{~b}$ show the calculated maps of optical absorption enhancement for TM and TE polarized illuminations, respectively, as a function of incident wavelength and nanowire period. The green, yellow and red regions in both maps correspond to an optical absorption enhancement (enhancement factor $>1$ ) for the nanowire anode-based OSC as compared with the one using commercial $150 \mathrm{~nm}$ thick ITO anode. For the TM illumination, the absorption enhancement can be attributed to the SPR effect inside the MIM OSC structure. In order to verify this point, we calculated the plasmon dispersion relation of the MIM structure by analytical method. The SP modes in a MIM structure with small thickness of the organic material layers $(-80 \mathrm{~nm}$ or less) would split into a symmetric mode and an antisymmetric mode, ${ }^{[26]}$ whose dispersion
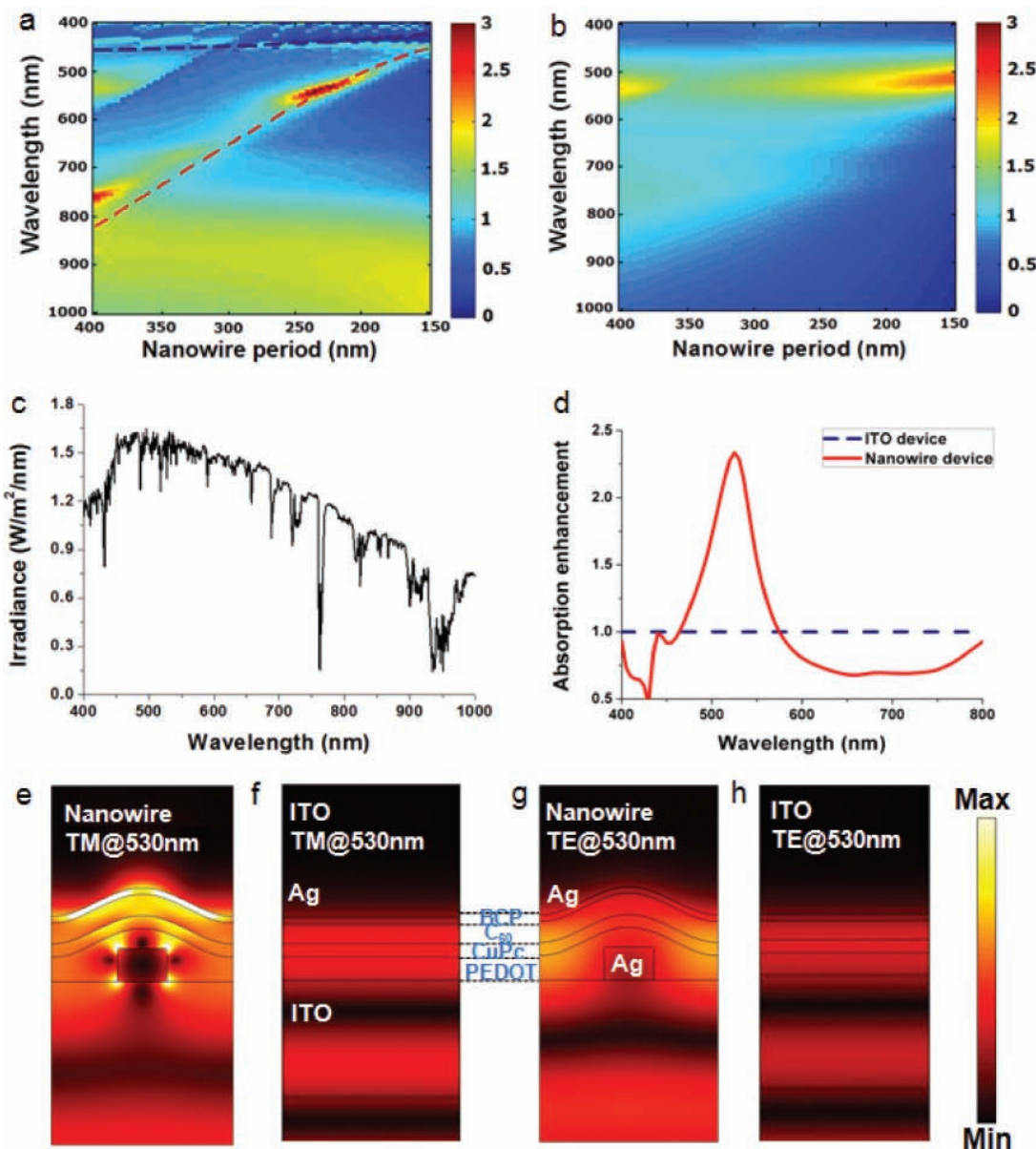

Figure 2. Calculated maps of optical absorption enhancement for nanowire device compared with ITO devices for (a) TM (b) TE polarized light. (c) Solar Irradiance for the standard AM1.5 spectrum. (d) Simulated optical absorption enhancement integrating TM and TE illuminations for nanowire device with $220 \mathrm{~nm}$ period. Simulated electric field profiles of nanowire and ITO devices at the wavelength $530 \mathrm{~nm}$ for (e) (f) TM illuminations, (g) (h) TE illuminations. All the profiles are in the same color scales. The thickness of PEDOT, CuPc, $\mathrm{C}_{60}$ and BCP layer is $30 \mathrm{~nm}$, $16 \mathrm{~nm}, 26 \mathrm{~nm}$ and $8 \mathrm{~nm}$, respectively. 
is in unpolarized, both TM and TE should be considered during the design of OSCs with optical enhancement effects.

Figure 2c shows the solar spectrum, where the irradiance maximum locates around $500-600 \mathrm{~nm}$. In this experiment, we choose the nanowire period as $220 \mathrm{~nm}$ so that the enhanced absorption range coincides with the peak solar spectrum. Though this wavelength range does not match the maximum absorption of the CuPc:C60 organic layers, we anticipate that a spectrally resolved incident photon to current efficiency (IPCE) measurement will reveal the enhancement effect as it is directly proportional to optical absorption efficiency of the organic semiconductors and excludes the absorption by the electrode. The simulated optical absorption enhancement for $220 \mathrm{~nm}$ nanowire period is given in Figure 2d, where the peak enhancement factor integrating TM and TE components reaches about 2.5 fold around the wavelength of $530 \mathrm{~nm}$ as compared to the control OSC with ITO anode. Figure $2 \mathrm{e}-\mathrm{h}$ give the electric field profiles of nanowire and ITO devices with TM and TE illuminations at the wavelength of $530 \mathrm{~nm}$, respectively. For both TM and TE illuminations, higher field intensity in the organic layers was obtained in the nanowire device than in the ITO device. For the TM illumination, the maximum fields concentrate near the edges of the bottom and top Ag electrodes, which is consistent with the field profiles of SP antisymmetric modes. ${ }^{[26]}$ On the other hand, TE illumination has the maximum fields between the cathode and nanowire anode, which also agrees with our above analysis of coupling to the waveguide mode. High optical field intensity in the organic layers results in high optical absorption, therefore we anticipate that this enhanced optical field by SPR and waveguide effects will lead to the enhancement of the solar cell efficiency.

To prove this principle, small molecular organic solar cells were fabricated using the organic materials as shown in Figure 1a and $1 \mathrm{~b}$. The Ag nanowires and a $70 \mathrm{~nm}$ thick continuous $\mathrm{Ag}$ layer were used as an anode and cathode, respectively. For comparison, devices with same thickness of organic layers but using ITO anode are also fabricated. The Ag nanowire electrodes are fabricated using nanoimprint lithography (NIL). ${ }^{[28]}$ The average transmittance of the $\mathrm{Ag}$ nanowire and ITO electrode samples (both $\sim 2.5 \mathrm{~cm} \times 2.5 \mathrm{~cm}$ in size) in the visible range is $77 \%$ and $87 \%$, respectively (Figure 3b). The nanowire is less transparent than ITO as expected, and the reflection from the nanowire makes the sample look more yellowish (Figure 3a).

Figure $3 d$ gives the measured $J-V$ characteristics for the nanowire and ITO devices with $80 \mathrm{~nm}$ thick organic layers. The open circuit voltage $\left(\mathrm{V}_{\text {oc }}\right)$ of the ITO device, $\sim 0.53 \mathrm{~V}$, was slightly greater than that of $\mathrm{Ag}$ nanowire devices, $\sim 0.5 \mathrm{~V}$. The fill-factors (FF) for both devices are about 0.5 . While having similar $V_{\text {oc }}$ and $\mathrm{FF}$, the short circle current $\left(J_{\mathrm{sc}}\right)$ showed a dramatic difference between the two devices. The $J_{\mathrm{sc}}$ of the nanowire device, $\sim 5.21 \mathrm{~mA} / \mathrm{cm}^{2}$, is about $40 \%$ higher than that of ITO device $\sim 3.72 \mathrm{~mA} / \mathrm{cm}^{2}$. Therefore the overall PCE of the nanowire device $(-1.32 \%)$ is enhanced about $35 \%$ than that of the ITO device $(\sim 0.96 \%)$.

In order to find the conclusive reason for the increased $J_{\mathrm{sc}}$, IPCE was measured for each device. The obtained external quantum efficiency (EQE) spectra of each device are shown in inset of Figure 3e. It can be clearly seen that the EQE of nanowire device is higher than that of ITO device across the whole visible range, which can explain the higher $J_{\mathrm{sc}}$ observed in the nanowire device. The enhancement in EQE between the nanowire device and the ITO device as a function of the wavelength can be extracted from the ratio of the two EQE curves, and is plotted in Figure 3e. A 2.5 fold EQE enhancement around the wavelength of $560 \mathrm{~nm}$ can be clearly observed, which compares favorably with the simulated absorption enhancement shown in Figure 2d. Since the EQE depends proportionally on the absorption efficiency of the active organic semiconductor layers and excludes the absorption effect by the Ag electrodes, we can attribute the observed enhancement of $J_{\text {sc }}$ to the enhanced optical absorption caused by the SPR and waveguide effects in the Ag nanowire device. There is another possibility which could also increase the efficiency due to the increase of the device surface area observed in the SEM image in Figure 1b. The difference between the peak and the valley in the sinusoidal shape of the device is about $30 \mathrm{~nm}$ which is estimated to increase the surface area by about $6 \%$. The increase of the device is not significant compared to the observed enhancement of the $J_{\mathrm{sc}}$. Therefore, we can conclude that the observed efficiency enhancement is mostly from the SPR and waveguide effects in the Ag nanowire device with very small if any contribution from the increase of surface area.

Next, several devices with different thicknesses of the organic layers were fabricated to investigate the dependence of $J_{\mathrm{sc}}$ enhancement on the organic layer thickness. For all cases, the thickness of the PEDOT and BCP was fixed at $30 \mathrm{~nm}$ and $8 \mathrm{~nm}$, respectively. The total organic layer thickness was varied by changing the thickness of $\mathrm{CuPc}$ and $\mathrm{C}_{60}$ while keeping their ratio the same. The thickness of $\mathrm{CuPc}$ and $\mathrm{C}_{60}$ for each device is summarized in inset table of Figure 4. The device parameters such as $V_{\text {oc }}$ and FF were similar for both the ITO and Ag devices at each organic layer thickness except device \#5. Device \#5 with Ag electrodes having the thinnest organic layer $(70 \mathrm{~nm})$ showed much reduced $V_{\text {oc }} \sim 0.37 \mathrm{~V}$, which is attributed to the shunt path between the Ag nanowires and the top cathode due to the very thin organic layers. For other devices, the $V_{\text {oc }}$ of the ITO device, $\sim 0.53 \mathrm{~V}$, was slightly greater than that of Ag devices, $\sim 0.5 \mathrm{~V}$. The FF of all devices was also similar for both ITO and Ag devices at each organic layer thickness as expected for bilayer structures. For device \#1 and \#2 having relatively thicker organic layers, the FF was no more than 0.4 for both ITO and Ag devices. On the other hand, the FF was about 0.5 for the device \#3 and \#4. Thicker organic layer presents higher resistance to the photocurrent due to low carrier mobility of organic materials. Similar $V_{\text {oc }}$ and FF for both ITO and Ag devices indicate that the patterned Ag electrode did not affect the device fabrication and performance. While having similar $V_{\mathrm{oc}}$ and FF for all devices, the $J_{\mathrm{sc}}$ showed a difference among different devices. Overall, the $J_{\text {sc }}$ of the ITO device decreases with reducing organic layer thickness, which is expected because of the reduced absorption efficiency in thinner devices. For thicker organic layers (device \#1 and \#2), the $J_{\mathrm{sc}}$ of the nanowire device is comparable to that of the ITO device. But for organic layers thinner than $100 \mathrm{~nm}$ (device $\# 3$, \#4, and \#5), the $J_{\mathrm{sc}}$ of the nanowire device was enhanced. The behavior of nanowire device's $J_{\mathrm{sc}}$ increasing with reduced organic layer thickness forms a sharp contrast to the ITO control device, and showed a maximum $J_{\mathrm{sc}}$ for $80 \mathrm{~nm}$ organic layer 
a

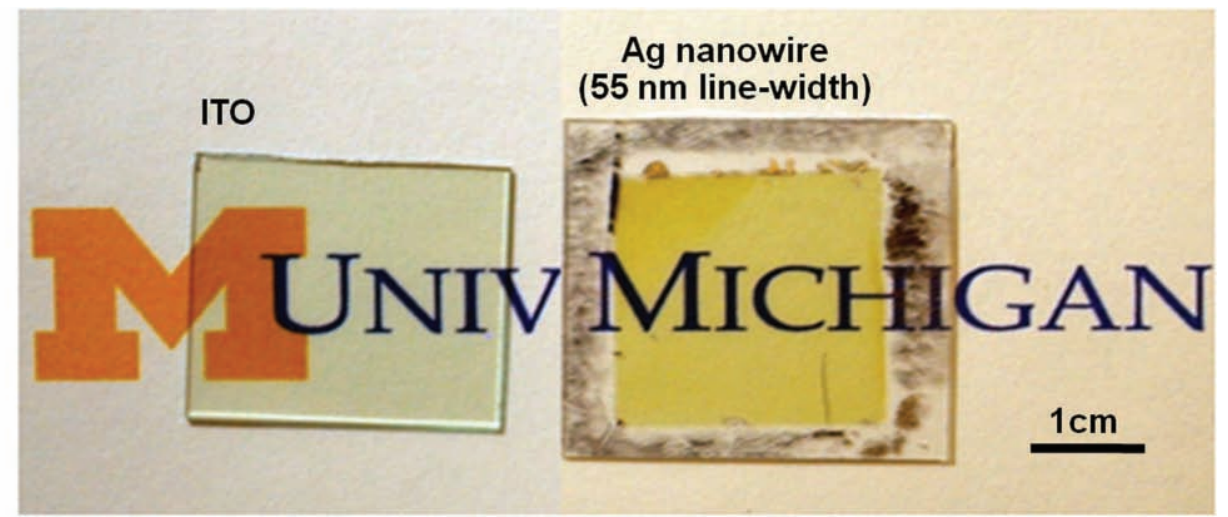

b
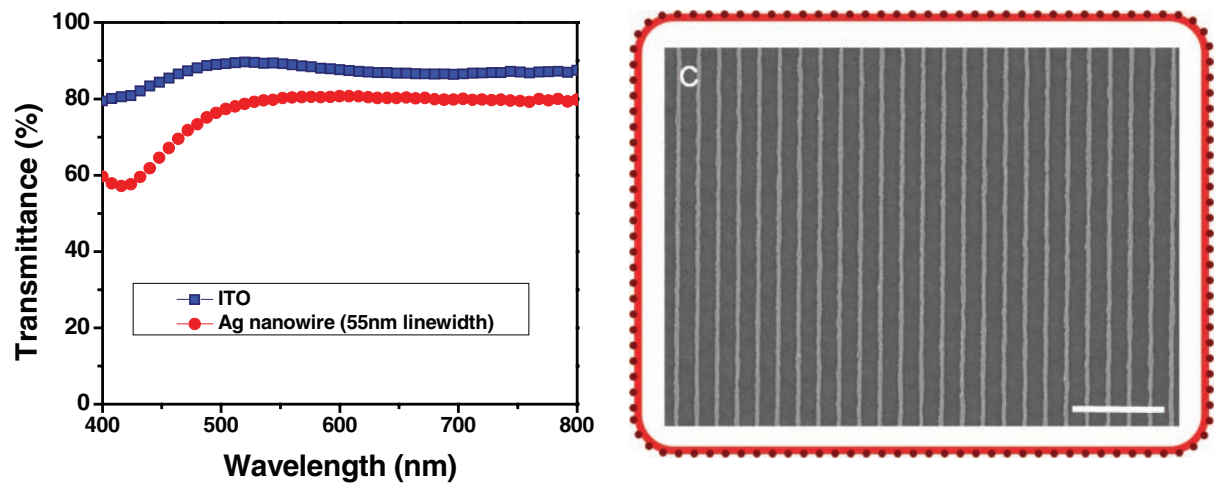

d
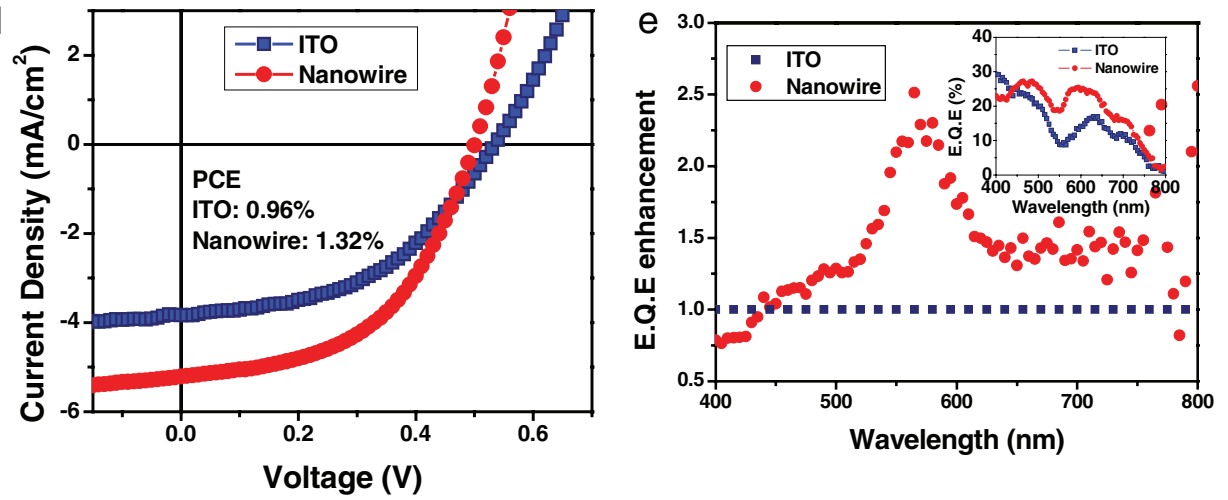

Figure 3. Photograph of the ITO-coated glass substrate and Ag nanowires with line-width of $55 \mathrm{~nm}$, from left to right. Scale bar is $1 \mathrm{~cm}$. (b) Transmittance spectra of ITO and Ag nanowire electrodes. (c) A scanning electron micrograph of Ag nanowire array with line-width of $55 \mathrm{~nm}$. The scale bar is $1 \mu \mathrm{m}$. (d) $J-V$ curve of the nanowire and control ITO devices. (e) External quantum efficiencies (EQE) enhancement of nanowire device with reference to ITO device. The inset gives the measured EQE of the nanowire and ITO devices.

thickness, as plotted in Figure 4. When the thickness of the total organic layer decreases to $70 \mathrm{~nm}$ the $J_{\mathrm{sc}}$ was increased further reaching an enhancement of $\sim 43 \%$ as compared to that of ITO device. However, due to lower $V_{\text {oc }}$, device $\# 5$ did not show higher PCE. Since the SPR effect in a MIM structure also depends on the dielectric layer thickness, ${ }^{[26]}$ this indicates that $80 \mathrm{~nm}$ organic layer thickness, combined with $220 \mathrm{~nm}$ nanowire period, provides the most efficient light coupling effect around the wavelength of peak solar irradiance.

In summary, we demonstrated organic solar cells with transparent $\mathrm{Ag}$ nanowire electrodes that can enhance the power conversion efficiency by surface plasmon resonance and waveguide effects. The $J_{\mathrm{sc}}$ was enhanced as much as $40 \%$ compared to that of the ITO device; and the EQE was enhanced by 2.5 fold at a wavelength of around $560 \mathrm{~nm}$ for an $80 \mathrm{~nm}$ thick organic layer. Due to this enhancement, the overall power conversion efficiency of the Ag device was enhanced by about 35\% compared to that of the ITO device under unpolarized light illumination. The PCE can be further enhanced by choosing organic semiconductors and adjusting the period of the Ag nanograting such that SP enhanced spectral range matches the peak absorption of the organic semicondcutors. The nanowires 


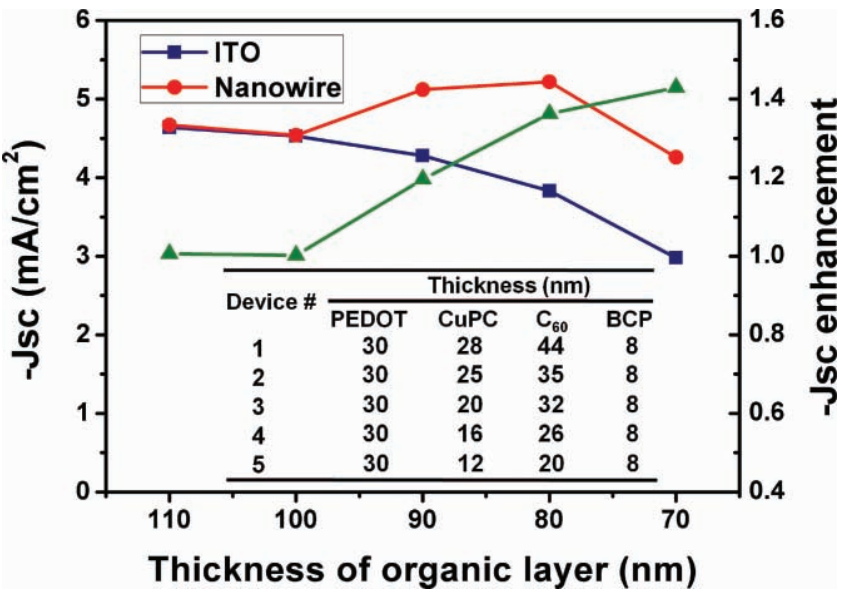

Figure 4. $J_{s c}$ versus total organic layer thickness. Several devices were fabricated and characterized with different total organic thickness ranging from $110 \mathrm{~nm}$ to $70 \mathrm{~nm}$ with $10 \mathrm{~nm}$ step, which correspond to the device number from 1 to 5 . The thickness of the PEDOT and BCP was fixed at $30 \mathrm{~nm}$ and $8 \mathrm{~nm}$, respectively, for all fabricated devices. The total organic layers thickness was controlled by changing the thickness of CuPc and $\mathrm{C}_{60}$ as shown in inset table.

can also be fabricated on flexible substrate by using a transfer printing technique ${ }^{[24]}$ and can be potentially extended to largearea high-throughput fabrication by a roll-to-roll process. ${ }^{[29]}$

\section{Experimental Section}

Fabrication of Ag nanwire electrodes: Ag nanowire electrode on glass substrates was fabricated with conventional thermal nanoimprint lithography (NIL) and shadow evaporation technique. NIL was performed in Nanonex NX2000 naonoimprinter (Princeton, NJ) using a mold with a period of $220 \mathrm{~nm}$, a line-width of $100 \mathrm{~nm}$, and a depth of $100 \mathrm{~nm}$ on a $100 \mathrm{~nm}$ thick MRI-8030 resist (Microresist Technology $\mathrm{GmbH}$ ) spin-coated on glass substrates, at a pressure of $600 \mathrm{psi}$ ( $1 \mathrm{psi} \cong 6.89 \mathrm{kpa}$ ) and a temperature of $180^{\circ} \mathrm{C}$ for $5 \mathrm{~min}$. The size of the mold and the glass substrate is about $2.5 \mathrm{~cm} \times 2.5 \mathrm{~cm}$. After NIL, the resist pattern has the opposite shape of the mold with a residual resist thickness of about $60 \mathrm{~nm}$, which is removed using oxygen RIE at $20 \mathrm{sccm}$ for $40 \mathrm{~s}$, at a power of $30 \mathrm{~W}$ and a pressure of $12 \mathrm{mTorr}$ ( $1 \mathrm{mTorr} \cong 133 \mathrm{mPa}$ ) after shadow evaporation of Ti on each sidewall of the resist pattern. $25 \mathrm{~nm}$ thick Ti was deposited on each sidewall of the imprinted grating structure to fabricate $\mathrm{Ag}$ nanowire array with a linewidth of about $55 \mathrm{~nm}$. Deposition of $40 \mathrm{~nm}$ thick Ag with $1 \mathrm{~nm} \mathrm{NiCr}$ using electron beam evaporation and lift-off completed the fabrication of the $\mathrm{Ag}$ grating electrode on a glass substrate.

Fabrication of organic solar cells (OSCs): To prove the enhancement of the efficiency of OSCs via surface plasmon resonance in Ag nanowire structures, organic bilayer heterojunction solar cells were fabricated using copper phthalocyaine (CuPc) and $\mathrm{C} 60$. Other thin film solar cells such as polymer bulk heterojuntion device using poly(3-hexylthophene) (P3HT) and [6,6]-phenyl $\mathrm{C}_{61}$ butyric acid methyl ester (PCBM), one of the most promising polymer semiconductors until now, can also be used to enhance the device efficiency after solving the compatibility issue of $\mathrm{Ag}$ cathode with those materials. For the fabrication of proposed device, the $\mathrm{Ag}$ nanowire electrodes and ITO on glass were firstly cleaned in acetone and isopropyl alcohol (IPA) under sonication for 30 min each. ITO coated glass had an additional 5 min UV ozone cleaning. Filtered PEDOT:PSS solution was spin-coated onto the $\mathrm{Ag}$ grating electrodes and ITO at $1000 \mathrm{rpm}$ for $30 \mathrm{~s}$, producing a $30 \mathrm{~nm}$ thick layer, and then baked at $120{ }^{\circ} \mathrm{C}$ for $15 \mathrm{~min}$ inside a homemade $\mathrm{N}_{2}$ purged box. To increase the wetting on the substrate and to control the PEDOT:PSS thickness, $0.2 \mathrm{wt} . \%$ Silquest 187 and $100 \mathrm{wt} . \%$ deionized water were added to the PEDOT (Baytron PH 500). Samples were then brought to an evaporation chamber from the $\mathrm{N}_{2}$ box. Thermal evaporation of the CuPc, $\mathrm{C}_{60}, \mathrm{BCP}$, and $70 \mathrm{~nm}$ thick $\mathrm{Ag}$ completed the organic solar cell fabrication. A shadow mask (diameter of $1 \mathrm{~mm}$ ) was put on the sample before the $\mathrm{Ag}$ evaporation. $\mathrm{CuPc}, \mathrm{C}_{60}$ and $\mathrm{BCP}$ were purchased from Sigma Aldrich and used as received.

Measurement of OSCs: I-V characteristics were measured with HP4156B semiconductor analyzer by illuminating OSCs with AM1.5 G simulated sun light using Oriel Solar Simulation at the irradiation intensity of $100 \mathrm{~mW} \mathrm{~cm}$, which was calibrated by power meter (OPHIR, Nova-Oriel) and a reference silicon solar cell. Incident photon to current efficiency (IPCE) spectra for EQE were measured with a $150 \mathrm{~W}$ Oriel Solar Simulator light source, an Acton ResearchCorp., Spectra-Pro 275 monochromator, a chopper wheel, and a Stanford Research 830 Lock-In Amplifier. The photocurrents were measured and normalized against a Hammamatsu Si photodiode reference, and long pass filters were used to block transmission of any stray $2^{\text {nd }}$ or $3^{\text {rd }}$ order diffractions outside of the range of interest.

\section{Acknowledgements}

M.-G.K. and T.X. contributed equally to this work. This work was supported in part by KACST. H.J.P. was supported in part by the Center for Solar and Thermal Energy Conversion, an Energy Frontier Research Center funded by the U.S. Department of Energy, Office of Science, Office of Basic Energy Sciences under Award Number DE-SC0000957. T.X. acknowledges support by the CSC scholarship. X. G. L. acknowledges support from NSFC 60825405 . L. J. G. acknowledges the travel support provided by the K. C. Wong Education Foundation, Hong Kong.

Received: April 18, 2010

Revised: June 1, 2010

Published online: August 23, 2010

[1] C. W. Tang, Appl. Phys. Lett. 1986, 48, 183.

[2] J. Y. Kim, K. Lee, N. E. Coates, D. Moses, T.-Q. Nguyen, M. Dante, A. J. Heeger Science 2007, 317, 222.

[3] S. H. Park, A. Roy, S. Beaupre, S. Cho, N. Coates, J. S. Moon, D. Moses, M. Leclerc, K. Lee, A. J. Heeger, Nat. Photon. 2009, 3, 297.

[4] D. Gilles, C. S. Markus, J. B. Christoph, Adv. Mater. 2009, 21, 1323.

[5] P. Peumans, A. Yakimov, S. R. Forrest, J. Appl. Phys. 2003, 93, 3693.

[6] S.-B. Rim, S. Zhao, S. R. Scully, M. D. McGehee, P. Peumans, Appl. Phys. Lett. 2007, 91, 243501.

[7] C. Cocoyer, L. Rocha, L. Sicot, B. Geffroy, R. de Bettignies, C. Sentein, C. Fiorini-Debuisschert, P. Raimond, Appl. Phys. Lett. 2006, 88, 133108 .

[8] D.-H. Ko, J. R. Tumbleston, L. Zhang, S. Williams, J. M. DeSimone, R. Lopez, E. T. Samulski, Nano Lett. 2009, 9, 2742.

[9] P. R. Barry, P. Peter, R. F. Stephen, J. Appl. Phys. 2004, 96, 7519.

[10] T. D. Heidel, J. K. Mapel, M. Singh, K. Celebi, M. A. Baldo Appl. Phys. Lett. 2007, 91, 093506.

[11] J. K. Mapel, M. Singh, M. A. Baldo, K. Celebi Appl. Phys. Lett. 2007, 90, 121102.

[12] A. J. Morfa, K. L. Rowlen, T. H. Reilly III, M. J. Romero, J. van de Lagemaat Appl. Phys. Lett. 2008, 92, 013504.

[13] T. H. Reilly III, J. van de Lagemaat, R. C. Tenent, A. J. Morfa, K. L. Rowlen Appl. Phys. Lett. 2008, 92, 243304.

[14] N. C. Lindquist, W. A. Luhman, S.-H. Oh, R. J. Homes Appl. Phys. Lett. 2008, 93, 123308. 
[15] X. Luo, T. Ishihara, Appl. Phys. Lett. 2004, 84, 4780.

[16] N. Fang, H. Lee, C. Sun, X. Zhang, Science 2005, 308, 534.

[17] M. G. Albrecht, J. A. Creighton, J. Am. Chem. Soc. 1977, 99, 5215.

[18] J. R. Sheats, D. B. Roitman, Synth. Met. 1998, 95, 79.

[19] W. S. Jahng, A. H. Francis, H. Moon, J. I. Nanos, M. D. Curtis, Appl. Phys. Lett. 2006, 88, 093504.

[20] B. Maennig, J. Drechsel, D. Gebeyehu, P. Simon, F. Kozlowski, A. Werner, F. Li, S. Grundmann, S. Sonntag, M. Koch, K. Leo, M. Pfeiffer, H. Hoppe, D. Meissner, N. S. Sariciftci, I. Riedel, V. Dyakonov, J. Parisi, Appl. Phys. A 2004, 79, 1.

[21] Y.-H. Kim, S.-H. Lee, J. Noh, S.-H. Han, Thin Solid Films 2006, 510, 305.
[22] M.-G. Kang, L. Guo, Adv. Mater. 2007, 19, 1391.

[23] M.-G. Kang, M.-S. Kim, J. Kim, L. Guo, Adv. Mater. 2008, 20, 4408

[24] M.-G. Kang, H. J. Park, S. H. Ahn, L. Guo, J. Sol. Energy Mater. Sol. Cells 2010, 94, 1179.

[25] M. G. Moharam, D. A. Pommet, E. B. Grann, T. K. Gaylord, J. Opt. Soc. Am. A 1995, 12, 1077.

[26] J. A. Dionne, L. A. Sweatlock, H. A. Atwater, Phys. Rev. B 2006, 73, 035407.

[27] R. A. Pala, J. White, E. Barnard, J. Liu, M. L. Brongersma, Adv. Mater. 2009, 21, 3504

[28] L. J. Guo, Adv. Mater. 2007, 19, 495.

[29] S. H. Ahn, L. Guo, ACS Nano 2009, 3, 2304. 